\title{
Cognitive responses to hypobaric hypoxia: implications for aviation training
}

This article was published in the following Dove Press journal:

Psychology Research and Behavior Management

I0 November 2014

Number of times this article has been viewed

\author{
Christopher Neuhaus ${ }^{1,2}$ \\ Jochen Hinkelbein ${ }^{2,3}$ \\ 'Department of Anesthesiology, \\ Heidelberg University Hospital, \\ Ruprecht Karls University of \\ Heidelberg, Heidelberg, ${ }^{2}$ Emergency \\ Medicine and Air Rescue Working \\ Group, German Society of \\ Aviation and Space Medicine \\ (DGLRM), Munich, ${ }^{3}$ Department of \\ Anesthesiology and Intensive Care \\ Medicine, University Hospital of \\ Cologne, Cologne, Germany
}

\begin{abstract}
The aim of this narrative review is to provide an overview on cognitive responses to hypobaric hypoxia and to show relevant implications for aviation training. A principal element of hypoxia-awareness training is the intentional evocation of hypoxia symptoms during specific training sessions within a safe and controlled environment. Repetitive training should enable pilots to learn and recognize their personal hypoxia symptoms. A time span of 3-6 years is generally considered suitable to refresh knowledge of the more subtle and early symptoms especially. Currently, there are two different technical approaches available to induce hypoxia during training: hypobaric chamber training and reduced-oxygen breathing devices. Hypoxia training for aircrew is extremely important and effective, and the hypoxia symptoms should be emphasized clearly to aircrews. The use of tight-fitting masks, leak checks, and equipment checks should be taught to all aircrew and reinforced regularly. It is noteworthy that there are major differences in the required quality and quantity of hypoxia training for both military and civilian pilots.
\end{abstract}

Keywords: cognitive response, aviation training, pilot, hypoxia, oxygen, loss of consciousness

\section{Introduction}

Ever since the first pioneer aviators took to the skies in hot-air balloons in the late eighteenth century, the human body has been subjected to various stressors and sources of danger, among others noise, vibration, thermal stress, acceleration, fatigue, radiation, and spatial disorientation. ${ }^{1}$ But of all the perils the human organism is exposed to after slipping the surly bonds of earth, the most hazardously perceived threat of flight at high altitude remains hypoxia. ${ }^{2}$

This is aggravated by the fact that with the exception of rare cases of carbon monoxide poisoning from reciprocating engines, hypoxia is the only condition presenting itself without clear and concise or conceivable symptoms, and no consistent physiologic reaction heralds the onset of further incapacitation. Moreover, where other stressors have a direct causal relationship to medical incapacitation and subsequent fatal accidents in aviation (eg, $g$-induced loss of consciousness), few accidents are known where hypoxia could be clearly identified as exclusive cause. ${ }^{3,4}$

Certainly unforgotten is the case of professional golfer Payne Stewart, who died on October 25, 1999 when the Learjet 35 carrying him and five others crashed in South Dakota after running out of fuel while everybody onboard was unconscious due to hypoxia after a sudden loss of cabin pressure. This flight covered 1,500 nautical miles $(2,778 \mathrm{~km})$ on autopilot while being escorted by US Air 
Force jets after radio contact was lost. ${ }^{5}$ A similar accident occurred in Greece in 2005, when a Helios Airways 737-300 crashed after running out of fuel with every soul on board believed incapacitated due to an erroneous setting of the pressurization system.

However the dramatic reports of "ghost planes" are hyped in mainstream media, it is the innumerable cases of accidents with multiple causes in which hypoxia is a key contributing factor that pose one of the greatest threats to aviation safety in general and explain the need for special aircrew hypoxiaawareness training.

This narrative review aims to outline the current state of scientific knowledge and derived concepts for hypoxia training, as well as implications for aviation training of pilots. The content of this review is limited to hypobaric hypoxia.

To identify relevant publications, we searched PubMed (http://www.pubmed.com) with the keywords "cognitive response", "hypobaric hypoxia", "aviation training", "implications", and "pilot training" in various combinations. Additionally, relevant related literature in PubMed and references of papers identified were analyzed.

\section{Hypobaric hypoxia effects}

It is generally accepted that the great potential for catastrophe and therefore grave danger of hypoxia lies in the variety and interindividual difference of symptoms, as well as the varying speed and order of onset relating to individual tolerance of low blood oxygen concentrations (partial pressure of oxygen in arterial blood). ${ }^{6-8}$

Hypoxia incidents occur most commonly at altitudes below 19,000 ft (5,791 m). ${ }^{9}$ Symptoms experienced include psychomotor impairment (including incoordination and tremors), impairment of cognitive function (concentration, confusion, memory loss, flexibility, working memory, and drowsiness), visual impairment (color intensity, light intensity, and blurred vision), psychological symptoms (anxiety, depression, and euphoria), shortness of breath, paresthesias, headache, dizziness, nausea, light-headedness, and tachycardia. ${ }^{2,8-11}$ As these authors stated, high altitude and consecutive hypobaric hypoxia also affect cognitive flexibility and anxiety state. ${ }^{10} \mathrm{Li}$ et al ${ }^{12}$ found that psychomotor performance was impaired. All hypoxia symptoms may occur at altitudes of $11,811 \mathrm{ft}(3,600 \mathrm{~m})$ and above. ${ }^{10}$

During sudden hypoxia exposure, impairment of memory is one of the most frequently reported symptoms, either during hypoxia-awareness training of aircrews or after an in-flight hypoxic incident. ${ }^{11}$ However, the effects of acute hypoxia on memory have only been sporadically studied in laboratory-controlled conditions for military aviation ${ }^{11}$ and even less for civilian aviation.

Therefore, a principal element of hypoxia-awareness training is the intentional evocation of hypoxia during training sessions within a safe and controlled environment to enable pilots to learn and recognize their personal symptoms.

\section{General training considerations}

Recent studies suggest that these symptoms remain largely consistent as experienced by an individual on repetitive exposure to acute hypoxia., ${ }^{2,6,8}$ Military aircrews around the world are routinely and repeatedly exposed to some kind of hypoxia-awareness training to learn what Smith described as one's "hypoxia signature". ${ }^{13}$ This training is usually repeated in fixed intervals, since studies indicate that individuals may experience a diminishing recall of symptoms over time; a time span 3-6 years is generally considered suitable to refresh knowledge of the more subtle and early symptoms especially, thereby creating a safety margin to the onset of more obvious symptoms of severe impairment. ${ }^{14}$ Moreover, this type of hypoxia-awareness training should be designed to include the proper use of emergency equipment and application of recovery procedures.

Since Bert (1878) and Barcroft (1925), studies on hypoxia have been conducted by lowering ambient oxygen partial pressure either through barometric pressure reduction (hypobaric hypoxia) or by lowering the $\mathrm{O}_{2}$ fraction (normobaric hypoxia). ${ }^{15}$ There are two different technical approaches currently in use to induce hypoxia during training, ie, hypobaric chamber training (HCT) to induce hypobaric hypoxia and normobaric nitrogen dilution using specialized devices (reduced-oxygen breathing devices [ROBDs]), to induce hypoxic hypoxia.

While all training protocols differ slightly in terms of medical supervision, general principals of cardiopulmonary monitoring are commonly used (including continuous electrocardiogram and blood oxygen saturation $\left[\mathrm{SpO}_{2}\right]$ monitoring) to ensure participant safety. Moreover, usually physicians or flight surgeons are present to supervise HCT. Hypobaric chambers in use commonly include one spot for medically trained staff on continuous $100 \%$ oxygen to offer immediate assistance should a medical emergency arise.

\section{Hypobaric chamber training}

In HCT, aircrews are usually exposed to altitudes of 25,000 ft $(7,620 \mathrm{~m})$. After a period of prebreathing $100 \%$ oxygen for denitrogenation to reduce the risk of decompression sickness (DCS), participants are subjected to reduced barometric 
pressure while wearing oxygen equipment and continuing to breathe $100 \% \mathrm{O}_{2}$ from a demand regulator and mask (commonly referred to as "mask on" simulation) while the chamber ascends to the preselected altitude.

After the desired altitude is reached, the mask is taken off, and participants are forced to breathe ambient air (ie, hypobaric hypoxia) while performing various psychomotor activities until symptoms of hypoxia occur. Thereafter, refitting the mask and switching to an emergency supply of $100 \%$ oxygen is the common challenge for the cognitively impaired participant. Usually, the training is combined with a demonstration of rapid decompression, the major setback being the increased risk for DCS and barotrauma. ${ }^{16,17}$

While the effectiveness of HCT has not been significantly challenged by the medical or aerospace physiology communities, recent studies have shown that as far as military incidents are concerned, hypoxia is more likely to occur in training aircraft or tactical jet platforms while an oxygen mask is being worn.

A study by Cable found that $63 \%$ of all hypoxia incidents in the Royal Australian Air Force over an 11-year period could be attributed to technical failures involving the onboard oxygen-delivery system. ${ }^{9}$ Older studies from the US Air Force reviewing hypoxia incidents from 1970 to 1980 showed similar results, acknowledging the fact that in $64.7 \%$ of all incidents, aircrew were using oxygen equipment and wearing oxygen masks while experiencing symptoms of hypoxia. ${ }^{18}$

Taken together, these findings challenge the effectiveness of a training paradigm in which experienced tactical jet aviators train to recognize hypoxia with their masks off. Cable noted that this group of aviators "may be better served by the use of an alternative hypoxia training method, whereby hypoxia is demonstrated while wearing an oxygen mask and breathing from a regulator". Such normobaric oxygendilution techniques "might better simulate the most likely hypoxia scenario facing an aviator operationally".

\section{Reduced-oxygen breathing training}

A new option for cost-effective and reasonably safe hypoxia training arose after researchers at the Naval Aerospace Medical Research Laboratory developed an ROBD that induces (hypoxic) hypoxia using mixed gas delivered through an aviator's oxygen mask. The ROBD simulates the rarified oxygen present at altitude by mixing air and nitrogen under normobaric conditions.

Early research demonstrated that the device could reliably reproduce the cognitive and physiological effects of hypoxia at sea level in humans. ${ }^{19,20}$ Furthermore, no significant difference between cognitive, physiological, and subjective effects of ROBD and HCT-induced hypoxia could be found. ${ }^{21}$

The development of ROBDs opened up countless possibilities in the further integration of hypoxia-awareness training into regular aircrew training. A study by Artino et al evaluated the feasibility of integrating hypoxia training using an ROBD with high-fidelity flight simulation, thereby integrating emergency and recovery flight training and medical awareness training in a platform-based simulation model (in the case of the study of the F/A-18 Hornet). ${ }^{22}$

A different approach has been the combination of both HCT and ROBD to compensate for the lack of pressure changes felt and experienced during normobaric ROBD training. This method, dubbed combined altitude and depleted oxygen (CADO), combines "exposure to moderate altitude of $10,000 \mathrm{ft}(3,048 \mathrm{~m})$ in a hypobaric chamber with the additional breathing of a gas mixture containing $10 \%$ oxygen and $90 \%$ nitrogen", as described by Singh et al. ${ }^{23}$ It was designed to "expose the subjects to a physiological altitude of $25,000 \mathrm{ft}$ $(7,620 \mathrm{~m})$, and to provide both a demonstration of hypoxia symptoms and the effects of pressure change".

Since training takes place well below the threshold of $18,000 \mathrm{ft}(5,486 \mathrm{~m})$ considered being a risk for DCS, ${ }^{13}$ this method is considered safer, while still exposing students to all relevant physiologic and subjective symptoms of hypoxia and rapid decompression. The authors found no difference in hypoxia symptoms experienced by test subjects between CADO and HCT, both in terms of symptom severity and frequency. Moreover, there was no significant difference during physiological and psychomotor-performance testing between the different hypoxia regimens. ${ }^{23}$ Obvious advantages of CADO hypoxia training include a reduced risk of DCS, no travel restrictions for participants after ascent in the hypobaric chamber, reduced training cost since instructors can also participate in more than one ascent every 24 hours, and the improved treatment options should a medical emergency occur during training.

\section{Discrepancies between the techniques}

Both techniques are feasible for aviation training, and offer individual advantages and disadvantages depending on the approach. Today, it is still being debated if the techniques result in different types of symptoms and changes in the human body. Since published studies are scarce and controversial, there exists no definitive answer to this question as of yet. A single study by Savourey et al described hypobaric 
hypoxia leading to greater hypoxemia, hypocapnia, blood alkalosis, and a lower $\mathrm{SpO}_{2}$ compared to normobaric hypoxia. The authors concluded that these physiological differences could be the consequence of an increase in dead-space ventilation, probably related to the barometric pressure reduction, and could be summed up under the term "the specific response to hypobaric hypoxia". ${ }^{15}$ Other authors also postulated that hypobaria instead of hypoxia per se plays an important role in the altered balance classically reported at altitude. ${ }^{24}$ However, detailed and conclusive knowledge cannot be derived from one individual study.

\section{Cognitive function and behavioral symptoms}

Impairment of cognitive flexibility is a major problem during hypobaric hypoxia, and significantly affects the ability to fly an aircraft. Xu et al ${ }^{10}$ tested 23 volunteers without prior hypoxic experience during simulated hypoxia at an altitude of 3,600 $\mathrm{m}(11,811 \mathrm{ft})$ in a hypobaric chamber. To quantify the hypoxia effect, the task-switch paradigm was used to explore cognitive flexibility in each phase, and the changing anxiety state was evaluated simultaneously. Reaction time (RT) showed a significant increase compared to the baseline value. Additionally, the anxiety level was higher during hypoxia. A remarkable negative correlation between anxiety level and RT was found. Therefore, the authors concluded that high altitude $(11,811 \mathrm{ft} / 3,600 \mathrm{~m})$ affects both cognitive flexibility and anxiety state. Anxiety before the hypoxia exposure improves cognitive flexibility performance, while anxiety after the hypoxia exposure hampers the performance because of the posthypoxia effect. ${ }^{10}$ With regard to the results of this study and the relevance for practical aviation training, it should be noted that hypoxia decreases RT. However, anxiety before hypoxia could improve cognitive flexibility.

In addition to the altered cognitive function during hypoxia, Li et al also found the mood state to be changed during hypoxia. ${ }^{12}$ In their study, the mood states of 18 healthy male volunteers were evaluated by self-assessment questionnaires Profile of Mood States and State Anxiety Inventory - after random exposure to simulated altitude of $300 \mathrm{~m}(984 \mathrm{ft}$, control), 2,800 m (9,186 ft), 3,600 m (11,811 ft), and 4,400 m $(14,435 \mathrm{ft})$ for 1 hour in a hypobaric chamber. Exposure to acute mild hypobaric hypoxia at 2,800 $\mathrm{m}(9,186 \mathrm{ft})$ for 1 hour had an adverse effect on the mood state, and the negative effect was further aggravated with increasing altitude.

During acute hypoxia exposure, impairment of memory is one of the most frequently reported symptoms, either during hypoxia-awareness training of aircrews or after a hypoxic incident in flight. However, the effects of acute hypoxia on memory function have scarcely been studied in laboratorycontrolled conditions. ${ }^{11}$ In regard to memory, both shortterm memory ${ }^{25}$ and working memory ${ }^{11}$ are affected during hypoxia. Moreover, none of these studies was performed in hypobaric conditions. ${ }^{11}$

One single and very interesting study in this field is from Malle et al. ${ }^{11}$ The authors analyzed 28 subjects (experimental group) who were exposed to a simulated altitude level of $10,000 \mathrm{~m}(31,000 \mathrm{ft})$ in a hypobaric chamber, while 29 subjects (control group) stayed at sea level. Working memory was assessed with the Paced Auditory Serial Addition Test (PASAT). Additionally, physiological parameters were recorded.

The main result was that working memory was strongly impaired in the hypoxic group and decreased linearly with hypoxemia, but $\mathrm{SpO}_{2}$ was weakly predictive of PASAT performance and vice versa. ${ }^{11}$ The authors concluded that working memory is of utmost importance in aircraft piloting. The PASAT, in association with $\mathrm{SpO}_{2}$ and electroencephalography recordings, could improve both hypoxia training and our understanding of the effects of hypoxia on memory. ${ }^{11}$

Besides cognitive function and memory, psychomotor performance is also paramount for safely piloting an aircraft and analyzing hypoxia effects. Li et $\mathrm{al}^{12}$ investigated 18 healthy male volunteers who performed finger tapping, simple RT (SRT) and four-choice RT (CRT) tests at simulated altitudes between $300 \mathrm{~m}$ (984 ft, control) and 4,400 m $(14,435 \mathrm{ft})$ in a hypobaric chamber. With increasing altitudes, the mean CRT was prolonged, and performance decreased compared with baseline value $(P<0.05)$, and performance decreased further at 4,400 $\mathrm{m}(14,435 \mathrm{ft}, P<0.01)$. No significant difference was found in finger tapping and SRT during exposure to $4,400 \mathrm{~m}(14,435 \mathrm{ft})$ for 1 hour. ${ }^{12}$ The results from this study demonstrated that there is no measurable impairment of visual RT or psychomotor performance during exposure to an altitude of 2,800 m (9,186 ft) for 1 hour. However, adverse effects on psychomotor performance were observed from 3,600 $\mathrm{m}(11,811 \mathrm{ft})$ and above. ${ }^{12}$

\section{Military training}

Due to the nature of its various missions, the military employs a wide variety of different rotary and fixed-wing aircraft, each with its own peculiar flight envelope. Helicopters mostly operate in low-to-medium altitudes (below 10,000 ft $[3,048 \mathrm{~m}])$ at moderate speed, where no supplemental oxygen is routinely administered, while jet aircraft can reach hostile atmospheric conditions well above $25,000 \mathrm{ft}(7,620 \mathrm{~m})$ at 
supersonic speed. Cockpits of transport aircraft are usually fully pressurized, while trainer and fighter aircraft are only partially pressurized or rely solely on oxygen masks. Careful consideration has to be taken in order to match hypoxia training to the specific type of mission flown with each airframe.

With over six decades of military jet operations and years of experience with HCT, there are ample data on cognitive impairment due to hypobaric hypoxia at altitude. Asmaro et $\mathrm{al}^{26}$ assessed performance in tasks relating to mental and behavioral processes using a variety of cognitive tests. They showed significant cognitive impairment, measured in RT and correct answers on the word-color Stroop test, at 25,000 ft $(7,620 \mathrm{~m})$. In the Stroop test, subjects are presented with the words red, yellow, blue, green, and white. Each word is presented in one of five colors: red, yellow, blue, green, or white. Subjects are told to respond to the actual color of the word and not the color that the word refers to. The combinations of word-color pairs are presented for a total of 30 seconds, and subjects receive a total score that is calculated by subtracting the number of correct responses from the number of errors. RT measurements are calculated by taking the number of correct responses and dividing by 30 . When these composite scores in each of the conditions are examined, RT changes caused by oxygen deprivation can be estimated. A lower number of correct answers indicates greater task impairment. The authors postulated that the results of their study suggest that these tests may be useful in screening for acute symptoms of hypoxia, and could provide insight into how discrete cognitive processes become impaired at various altitudes. ${ }^{26}$ Additionally, a French group - Malle et al ${ }^{11}$ - postulated that during military hypoxia training, the PASAT in association with pulse oximetry and electroencephalography recordings could improve both hypoxia training and the understanding of the effects of hypoxia on memory.

\section{Civilian training}

There are nearly no scientific data available on hypoxia training for civilian pilots or civilian pilot training. While there are several studies published for military aviation training on this topic, an extensive Medline search revealed no useful studies for civilian aviation training.

Since military facilities for hypobaric training are usually not accessible for nonmilitary personnel, these facilities are only infrequently used in civilian pilot training. In the few cases where larger civil aviation schools cooperate with military facilities, hypoxia awareness may be part of the curriculum. In private pilot training, however, hypoxia awareness remains an absolute exception despite being more than useful, since these pilots usually fly nonpressurized aircraft and are therefore at high risk for hypobaric hypoxia.

There is the sole option to prevent hypoxia by using supplemental oxygen. Depending on the type of masks and equipment, there is a wide variety in the oxygen flow required to prevent hypoxia. ${ }^{27}$ Therefore, proper fitting of masks, leak checks, and equipment checks should be taught to all aircrew and reinforced regularly, ${ }^{9}$ especially with private pilots.

\section{Conclusion}

For military aviation training, hypoxia training for aircrew is extremely important and effective. ${ }^{9}$ Since symptoms can occur even below 19,000 $\mathrm{ft}(5,791 \mathrm{~m})$, they should be emphasized clearly to aircrews, whose expectation may be that it is only a problem of high altitude. ${ }^{9}$ The use of tight-fitting masks, leak checks, and equipment checks should be taught to all aircrew and reinforced regularly, ${ }^{9}$ since these measures may reduce the incidence of artificial hypoxia significantly.

HCT methods simulating subtle incapacitation while wearing oxygen equipment should be further explored for a more realistic training approach. Finally, hypobaric hypoxia in flight remains a serious threat to aviators, and can result in fatalities. ${ }^{9}$

\section{Key points and practical implications}

- A principal element of hypoxia-awareness training is the intentional evocation of hypoxia symptoms during training sessions within a safe and controlled environment to enable pilots to learn and recognize their personal symptoms.

- This training is usually repeated in fixed intervals. A time span of 3-6 years is generally considered suitable to refresh knowledge of the more subtle and early symptoms especially.

- There are two different technical approaches currently in use to induce hypoxia during training, ie, HCT and ROBDs.

- There are major differences in the required quality and quantity of hypoxia training for both military and civilian pilots.

- Hypoxia training for aircrew is extremely important and effective, and hypoxia symptoms should be emphasized clearly to aircrews. This applies to both military and civilian pilots.

- The use of tight-fitting masks, leak checks, and equipment checks should be taught to all aircrew and reinforced regularly. 


\section{Disclosure}

The authors report no conflicts of interest in this work.

\section{References}

1. Engle E, Lott A. From Montgolfier to Stratolab. In: Paanen E, editor. Man in Flight: Biomedical Achievements in Aerospace. Annapolis (MD): Leeward; 1979:31-38.

2. Harding RM. Hypoxia and hyperventilation. In: Ernsting J, Nicholson AN, Rainford DJ, editors. Aviation Medicine. Oxford: Butterworth Heinemann; 1999:43-58.

3. Hinkelbein J. Medical incapacitation causing in-flight accidents: which evidence do we have? Aviat Space Environ Med. 2007;78:304.

4. Hinkelbein J, Dambier M, Glaser E, Landgraf H. Medical Incapacitation im Cockpit: Inzidenz, Ursachen und Folgen. Flugmed Tropenmed Reisemed. 2008;15:14-19.

5. Newman DG. Runaway plane. Flight Saf Aust. 2000;3-4:42-44.

6. Federal Aviation Administration Civil Aerospace Medical Institute. Hypoxia: The Higher You Fly ... The Less Air in the Sky. Oklahoma City: FAA Civil Aerospace Medical Institute; 2004.

7. Gradwell DP. Hypoxia and hyperventilation. In: Rainford DJ, Gradwell DP, editors. Ernsting's Aviation Medicine. 4th ed. London: Hodder Arnold; 2006:41-56.

8. Pickard JS. The atmosphere and respiration. In: Dehart RL, Davies JR, editors. Fundamentals of Aerospace Medicine. 3rd ed. Philadelphia: Lippincott Williams and Wilkins; 2002:19-38.

9. Cable GG. In-flight hypoxia incidents in military aircraft: causes and implications for training. Aviat Space Environ Med. 2003;74:169-172.

10. Xu L, Wu Y, Zhao T, et al. [Effect of high altitude hypoxia on cognitive flexibility]. Zhongguo Ying Yong Sheng Li Xue Za Zhi. 2014;30: 106-119. Chinese.

11. Malle C, Quinette P, Laisney M, et al. Working memory impairment in pilots exposed to acute hypobaric hypoxia. Aviat Space Environ Med. 2013;84:773-779.

12. Li XY, Wu XY, Fu C, Shen XF, Yang CB, Wu YH. Effects of acute exposure to mild or moderate hypoxia on human psychomotor performance and visual-reaction time. Space Med Med Eng (Beijing). 2000;13: 235-239.

13. Smith AM. Hypoxia symptoms in military aircrew: long-term recall vs acute experience in training. Aviat Space Environ Med. 2008;79:54-57.
14. Johnston BJ, Iremonger GS, Hunt S, Beattie E. Hypoxia training: symptom replication in experienced military aircrew. Aviat Space Environ Med. 2012;83:962-967.

15. Savourey G, Launay JC, Besnard Y, Guinet A, Travers S. Normo- and hypobaric hypoxia: are there any physiological differences? Eur J Appl Physiol. 2003;89:122-126.

16. Ohrui N, Takeuchi A, Tong A, et al. Physiological incidents during 39 years of hypobaric chamber training in Japan. Aviat Space Environ Med. 2002;73:395-398.

17. Smart TL, Gable GG. Australian Defence Force hypobaric chamber training, 1984-2001. ADF Health. 2004;5:3-10.

18. Rayman RB, McNaughton GB. Hypoxia: USAF experience 1970-1980. Aviat Space Environ Med. 1983;54:357-359.

19. Sausen KP, Bower EA, Stiney ME, Feigl C, Wartman R, Clark JB. A closed-loop reduced oxygen breathing device for inducing hypoxia in humans. Aviat Space Environ Med. 2003;74:1190-1197.

20. Sausen KP, Wallick MT, Slobodnik B, et al. The reduced oxygen breathing paradigm for hypoxia training: physiological, cognitive, and subjective effects. Aviat Space Environ Med. 2001;72:539-545.

21. Vacchiano CA, Vagedes K, Gonzalez D. Comparison of the physiological, cognitive, and subjective effects of sea level and altitude-induced hypoxia [abstract]. Aviat Space Environ Med. 2004;75:B56.

22. Artino AR, Folga RV, Swan BD. Mask-on hypoxia training for tactical jet aviators: evaluation of an alternate instructional paradigm. Aviat Space Environ Med. 2006;77:857-863.

23. Singh B, Cable GG, Hampson GV, Pascoe GD, Corbett M, Smith A. Hypoxia awareness training for aircrew: a comparison of two techniques. Aviat Space Environ Med. 2010;81:857-863.

24. Degache F, Larghi G, Faiss R, Deriaz O, Millet G. Hypobaric versus normobaric hypoxia: same effects on postural stability? High Alt Med Biol. 2012;13:40-45.

25. Du JY, Li XY, Zhuang Y, Wu XY, Wang T. [Effects of acute mild and moderate hypoxia on human short memory]. Space Med Med Eng (Beijing). 1999;12:270-273.

26. Asmaro D, Mayall J, Ferguson S. Cognition at altitude: impairment in executive and memory processes under hypoxic conditions. Aviat Space Environ Med. 2013;84:1159-1165.

27. Hinkelbein J, Glaser E, Doerrstein J, Genzwuerker HV. Oxygen delivery comparison of two constant-flow masks during flight to $6863 \mathrm{~m}$. Aviat Space Environ Med. 2006;77:540-544.
Psychology Research and Behavior Management

\section{Publish your work in this journal}

Psychology Research and Behavior Management is an international, peerreviewed, open access journal focusing on the science of psychology and its application in behavior management to develop improved outcomes in the clinical, educational, sports and business arenas. Specific topics covered include: Neuroscience, memory \& decision making; Behavior
Dovepress

modification \& management; Clinical applications; Business \& sports performance management; Social and developmental studies; Animal studies. The manuscript management system is completely online and includes a quick and fair peer-review system. Visit http://www.dovepress. com/testimonials.php to read real quotes from published authors. 